\title{
ON THE WALL POLYNOMIALS AND THE L-M-W CONJECTURES
}

\author{
GEORGE E. ANDREWS
}

(Received 23 August 1982; revised 25 January 1983)

Communicated by W. D. Wallis

\begin{abstract}
This paper provides explicit formulas for the Wall polynomials which arise in Wall's work on conjugacy classes in the unitary, symplectic and orthogonal groups. From these explicit formulas are easily derived six interesting limiting identities including the two that arise in the Lusztig-MacdonaldWall conjectures.

1980 Mathematics subject classification (Amer. Math. Soc.): primary 33 A 30; secondary 10 A 45, 20 C 15.

Keywords and phrases: $q$-series, Gaussian polynomials, Wall polynomials, group theory.
\end{abstract}

\section{Introduction}

G. Lusztig in his pathbreaking paper on the representation theory of the finite classical groups [4] found that the following polynomials and associated limits were essential when dealing with the case of characteristic 2 .

Let us define polynomials $\chi_{n}=\chi_{n}(a, b, q)$ by $\chi_{-1}=a, \chi_{0}=b$ and for $n \geqslant 0$

$$
\begin{gathered}
\chi_{2 n+1}=\chi_{2 n}+q^{2 n+1} \chi_{2 n-1}, \\
\chi_{2 n+2}=\chi_{2 n+1}+q^{n+1}\left(1+q^{n+1}\right)\left(\chi_{2 n+1}+\left(1-q^{2 n+1}\right) \chi_{2 n-1}\right) .
\end{gathered}
$$

Now define $\chi(a, b, q)=\lim _{n \rightarrow \infty} \chi_{n}(a, b, q)$. Then it turns out that

$$
\chi(1,1, q)=\frac{\sum_{i=-\infty}^{\infty} q^{i^{2}}}{\prod_{j=1}^{\infty}\left(1-q^{j}\right)},
$$

This work was partially supported by a National Science Foundation grant.

C 1984 Australian Mathematical Society 0263-6115/84 \$A2.00+0.00 
and

$$
\chi(0,1, q)=\frac{\sum_{i=0}^{\infty} q^{i(i+1)}}{\prod_{j=1}^{\infty}\left(1-q^{j}\right)} .
$$

Identities (1.3) and (1.4) were first established in [2]. Subsequently A. Garsia and J. Remmel [3] made an extensive combinatorial study of the proof given in [2] and provided many insights and simplifications surrounding the development. However, both [2] and [3] tend to focus on the actual limit functions $\chi(a, b, q)$ and regard the polynomials $\chi_{n}(a, b, q)$ as means to this end.

The polynomials $\chi_{n}(a, b, q)$ were first defined by G. E. Wall [5], and so we have called them "Wall Polynomials". The object of this paper is to provide explicit formulas for the Wall polynomials which allow one to prove not only (1.3) and (1.4) (the L-M-W conjectures) but (3.5)-(3.8) as well. These explicit formulas are given in Theorem 1 whose proof occupies Section 2. Section 3 is devoted to the limiting results described above, and Section 4 discusses some obvious open questions.

IBM's symbolic manipulation language SCRATCHPAD was instrumental in the discovery of (3.5) - (3.8) and in the determination of (2.4)-(2.7). Indeed the ease with which (2.4)-(2.7) were discovered illustrates the great utility of SCRATCHPAD in problems of this type.

The following tables (produced by SCRATCHPAD) present the first few Wall polynomials:

$$
\begin{array}{lc} 
& \text { TABLE } 1 \\
n & x_{n}(1,1, q) \\
0 & 1 \\
1 & 1+q \\
2 & 1+3 q+2 q^{2} \\
3 & 1+3 q+2 q^{2}+q^{3}+q^{4} \\
4 & 1+3 q+4 q^{2}+5 q^{3}+5 q^{4}+4 q^{5}+2 q^{6} \\
5 & 1+3 q+4 q^{2}+5 q^{3}+5 q^{4}+5 q^{5}+5 q^{6}+2 q^{7}+q^{8}+q^{9} \\
6 & 1+3 q+4 q^{2}+7 q^{3}+11 q^{4}+11 q^{5}+13 q^{6}+14 q^{7}+11 q^{8}+9 q^{9}+6 q^{10}+4 q^{11}+2 q^{12} \\
7 & 1+3 q+4 q^{2}+7 q^{3}+11 q^{4}+11 q^{5}+13 q^{6}+15 q^{7}+14 q^{8}+13 q^{9}+11 q^{10} \\
7 & +9 q^{11}+7 q^{12}+5 q^{13}+2 q^{14}+q^{15}+q^{16}
\end{array}
$$

TABLE 2

$\begin{array}{lc}n & \chi_{n}(0,1, q) \\ 0 & 1 \\ 1 & 1+q+q^{2} \\ 2 & 1+q+q^{2}+q^{3} \\ 3 & 1+q+3 q^{2}+2 q^{3}+3 q^{4}+q^{5}+q^{6} \\ 4 & 1+q+3 q^{2}+2 q^{3}+3 q^{4}+2 q^{5}+2 q^{6}+q^{7}+q^{8} \\ 5 & 1+q+3 q^{2}+4 q^{3}+5 q^{4}+6 q^{5}+7 q^{6}+6 q^{7}+6 q^{8} \\ 6 & +4 q^{9}+3 q^{10}+q^{11}+q^{12} \\ & \\ 7 & 1+q+3 q^{2}+4 q^{3}+5 q^{4}+6 q^{5}+7 q^{6}+7 q^{7}+7 q^{8}+7 q^{9} \\ & +5 q^{10}+4 q^{11}+3 q^{12}+2 q^{13}+q^{14}+q^{15}\end{array}$




\section{The main theorem}

For the statement and proof of this theorem we require some simple facts about Gaussian polynomials:

$$
\left[\begin{array}{l}
A \\
B
\end{array}\right]= \begin{cases}\frac{\left(1-q^{A}\right)\left(1-q^{A-1}\right) \cdots\left(1-q^{A-B+1}\right)}{\left(1-q^{B}\right)\left(1-q^{B-1}\right) \cdots(1-q)}, & B \geqslant 0 \\
0, & B<0\end{cases}
$$

where $A$ is a nonnegative integer and $B$ is any integer.

These polynomials satisfy

$$
\begin{aligned}
{\left[\begin{array}{c}
A \\
B
\end{array}\right] } & =\left[\begin{array}{l}
A-1 \\
B-1
\end{array}\right]+q^{B}\left[\begin{array}{c}
A-1 \\
B
\end{array}\right] \\
& =\left[\begin{array}{c}
A-1 \\
B
\end{array}\right]+q^{A-B}\left[\begin{array}{c}
A-1 \\
B-1
\end{array}\right],
\end{aligned}
$$

from [1, page 35, equations (3.3.3) and (3.3.4)].

\section{THEOREM 1.}

$$
\begin{gathered}
\chi_{2 n-1}(1,1, q)=\sum_{j=-\infty}^{\infty}\left[\begin{array}{c}
2 n \\
n+2 j
\end{array}\right] q^{j^{2}}, \\
\chi_{2 n}(1,1, q)=\sum_{j=-\infty}^{\infty}\left(\left[\begin{array}{c}
2 n+1 \\
n+2 j+1
\end{array}\right]+q^{n}\left[\begin{array}{c}
2 n \\
n+2 j+1
\end{array}\right]\right) q^{j^{2}}, \\
\chi_{2 n-1}(0,1, q)=\sum_{j=0}^{\infty}\left[\begin{array}{c}
2 n \\
n-1-2 j
\end{array}\right] q^{j^{2}+j}, \\
\chi_{2 n}(0,1, q)=\sum_{j=0 \infty}^{\infty}\left[\begin{array}{l}
2 n+1 \\
n-2 j
\end{array}\right] q^{j^{2}+j}+\sum_{j=1}^{\infty}\left[\begin{array}{c}
2 n \\
n-2 j
\end{array}\right] q^{j^{2}+j} .
\end{gathered}
$$

Proor. We only show that the proposed representations of these polynomials actually satisfy the recurrences (1.1) and (1.2) together with the appropriate initial conditions. The theorem then follows by mathematical induction.

We treat (2.4) and (2.5) first. Define $P(n, m)$ by

$$
P(n, m)=\sum_{j=-\infty}^{\infty}\left[\begin{array}{c}
n+m \\
n+2 j
\end{array}\right] q^{j^{2}}
$$

Then by replacing $j$ by $-j$, we see that

$$
P(n, m)=P(m, n),
$$


and

$$
\begin{aligned}
P(n, m) & =\sum_{j=-\infty}^{\infty}\left(\left[\begin{array}{c}
n+m-1 \\
n+2 j-1
\end{array}\right]+q^{n+2 j}\left[\begin{array}{c}
n+m-1 \\
n+2 j
\end{array}\right]\right) q^{j^{2}} \\
& =P(n-1, m)+q^{n-1} \sum_{j=-\infty}^{\infty}\left[\begin{array}{c}
n+m-1 \\
n+2 j
\end{array}\right] q^{(j+1)^{2}} \\
& =P(n-1, m)+q^{n-1} \sum_{j=-\infty}^{\infty}\left[\begin{array}{c}
n+m-1 \\
n+2 j-2
\end{array}\right] q^{j^{2}} \\
& =P(n-1, m)+q^{n-1} P(n-2, m+1) .
\end{aligned}
$$

Now define

$$
X_{2 n-1}=P(n, n),
$$

and

$$
X_{2 n}=P(n+1, n)+q^{n} P(n+1, n-1) .
$$

Clearly $X_{-1}=X_{0}=1$, and

\section{Also}

$$
\begin{aligned}
X_{2 n+1} & =P(n+1, n+1) \\
& =P(n, n+1)+q^{n} P(n-1, n+2) \quad(\text { by }(2.10)) \\
& =P(n+1, n)+q^{n} P(n+2, n-1) \quad(\text { by }(2.9)) \\
& =P(n+1, n)+q^{n}\left(P(n+1, n-1)+q^{n+1} P(n, n)\right) \quad(\text { by }(2.10)) \\
& =X_{2 n}+q^{2 n+1} X_{2 n-1} .
\end{aligned}
$$

$$
\begin{aligned}
X_{2 n+2}=P(n+2, n+1)+q^{n+1} P(n+2, n) & \\
= & P(n+1, n+1)+q^{n+1} P(n, n+2)+q^{n+1} P(n+2, n) \quad(\text { by }(2.10)) \\
= & X_{2 n+1}+2 q^{n+1} P(n+2, n) \quad(\text { by }(2.9) \text { and }(2.11)) \\
= & X_{2 n+1}+2 q^{n+1}\left(P(n+1, n)+q^{n+1} P(n, n+1)\right) \quad(\text { by }(2.10)) \\
= & X_{2 n+1}+2 q^{n+1}\left(1+q^{n+1}\right) P(n+1, n) \quad(\text { by }(2.9)) \\
= & X_{2 n+1}+q^{n+1}\left(1+q^{n+1}\right) P(n+1, n) \\
& +q^{n+1}\left(1+q^{n+1}\right)\left(P(n, n)+q^{n} P(n-1, n+1)\right) \\
= & X_{2 n+1}+q^{n+1}\left(1+q^{n+1}\right) X_{2 n-1} \\
& +q^{n+1}\left(1+q^{n+1}\right)\left(P(n+1, n)+q^{n} P(n+1, n-1)\right) \quad(\text { by }(2.11) \text { and (2.9)) } \\
= & X_{2 n+1}+q^{n+1}\left(1+q^{n+1}\right)\left(X_{2 n}+X_{2 n-1}\right) \\
= & X_{2 n+1}+q^{n+1}\left(1+q^{n+1}\right)\left(X_{2 n+1}+\left(1-q^{2 n+1}\right) X_{2 n-1}\right) \quad(\text { by }(2.13)) .
\end{aligned}
$$


Thus the polynomials $X_{n}$ satisfy the initial conditions $X_{-1}=X_{0}=1$ and (1.1) and (1.2). Consequently

$$
X_{n}=\chi_{n}(1,1, q) \text { for all } n \geqslant-1 .
$$

Combining (2.15) with (2.11) and (2.12), we see that (2.4) and (2.5) are established.

For (2.6) and (2.7) we define

$$
Q_{a}(n, m)=\sum_{j \geq a}\left[\begin{array}{l}
n+m \\
n-2 j
\end{array}\right] q^{j^{2}+j}
$$

Now

$$
\begin{aligned}
Q_{a}(n, m) & =\sum_{j>a}\left(\left[\begin{array}{c}
n+m-1 \\
n-2 j-1
\end{array}\right]+q^{n-2 j}\left[\begin{array}{c}
n+m-1 \\
n-2 j
\end{array}\right]\right) q^{j^{2}+j} \\
& =Q_{a}(n-1, m)+q^{n} \sum_{j>a-1}\left[\begin{array}{c}
n+m-1 \\
n-2-2 j
\end{array}\right] q^{j^{2}+j} \\
& =Q_{a}(n-1, m)+q^{n} Q_{a-1}(n-2, m+1)
\end{aligned}
$$

and

$$
\begin{aligned}
Q_{a}(n, m)= & \sum_{j>a}\left(\left[\begin{array}{c}
n+m-1 \\
n-2 j
\end{array}\right]+q^{m+2 j}\left[\begin{array}{c}
n+m-1 \\
n-2 j-a 1
\end{array}\right]\right) q^{j^{2}+j} \\
& \times Q_{a}(n, m-1)+q^{m-2} \sum_{j \geq a+1}\left[\begin{array}{c}
n+m-1 \\
n+1-2 j
\end{array}\right] q^{j^{2}+j} \\
= & Q_{a}(n, m-1)+q^{m-2} Q_{a+1}(n+1, m-2)
\end{aligned}
$$

Next define

$$
Y_{2 n-1}=Q_{0}(n-1, n+1),
$$

and

$$
Y_{2 n}=Q_{0}(n, n+1)+q^{n} Q_{1}(n, n) .
$$

Clearly $Y_{-1}=0, Y_{0}=1$, and

$$
\begin{aligned}
Y_{2 n+1} & =Q_{0}(n, n+2) \\
& =Q_{0}(n, n+1)+q^{n} Q_{1}(n+1, n) \quad(\text { by }(2.18)) \\
& =Y_{2 n}-q^{n} Q_{1}(n, n)+q^{n} Q_{1}(n+1, n) \quad(\text { by }(2.20)) \\
& =Y_{2 n}+q^{2 n+1} Q_{0}(n-1, n+1) \quad(\text { by }(2.17)) \\
& =Y_{2 n}+q^{2 n+1} Y_{2 n-1}(q) \quad(\text { by }(2.19)) .
\end{aligned}
$$




\section{Finally}

$$
\begin{aligned}
& Y_{2 n+2}=Q_{0}(n+1, n+2)+q^{n+1} Q_{1}(n+1, n+1) \\
& =Y_{2 n+1}-Q_{0}(n, n+2)+Q_{0}(n+1, n+2)+q^{n+1} Q_{1}\left(n+1, n_{1}\right) \\
& =Y_{2 n+1}+q^{n+1} Q_{-1}(n-1, n+3)+q^{n+1} Q_{1}(n+1, n+1) \\
& =Y_{2 n+1}+q^{n+1}\left(\left[\begin{array}{c}
2 n+2 \\
n+1
\end{array}\right]+Q_{0}(n-1, n+3)\right. \\
& \left.\quad+Q_{0}(n+1, n+1)-\left[\begin{array}{c}
2 n+2 \\
n+1
\end{array}\right]\right)
\end{aligned}
$$

$$
\begin{aligned}
= & Y_{2 n+1}+q^{n+1}\left(Q_{0}(n-1, n+3)+Q_{0}(n+1, n+1)\right) \\
= & Y_{2 n+1}+\left(q^{n+1}+q^{2 n+2}\right) Y_{2 n-1} \\
& +q^{n+1}\left(-\left(1+q^{n+1}\right) Q_{0}(n-1, n+1)\right. \\
& \left.\quad+Q_{0}(n-1, n+3)+Q_{0}(n+1, n+1)\right)
\end{aligned}
$$$$
=Y_{2 n+1}+\left(q^{n+1}+q^{2 n+2}\right)\left(Y_{2 n-1}+Y_{2 n}\right)
$$$$
+q^{n+1}\left(-\left(1+q^{n+1}\right) Q_{0}(n-1, n+1)\right.
$$$$
-\left(1+q^{n+1}\right)\left(Q_{0}(n, n+1)+q^{n} Q_{1}(n, n)\right)
$$$$
\left.+Q_{0}(n-1, n+3)+Q_{0}(n+1, n+1)\right)
$$

$$
\begin{aligned}
&=Y_{2 n+1}+\left(q^{n+1}+q^{2 n+2}\right)\left(Y_{2 n+1}+Y_{2 n}\right) \\
&+q^{n+1}\left(-1\left(1+q^{n+1}\right)\right. Q_{0}(n-1, n+1)-\left(1+q^{n+1}\right) \\
& \cdot\left(Q_{0}(n, n+1)+q^{n} Q_{1}(n, n)\right)+Q_{0}(n-1, n+2) \\
&\left.\quad+q^{n+1} Q_{1}(n, n+1)+Q_{0}(n+1, n+1)\right)
\end{aligned}
$$

$$
\begin{aligned}
=Y_{2 n+1}+ & \left(q^{n+1}+q^{2 n+2}\right)\left(Y_{2 n-1}+Y_{2 n}\right) \\
+q^{n+1}( & -\left(1+q^{n+1}\right) Q_{0}(n-1, n+1)-\left(1+q^{n+1}\right) \\
& \cdot\left(Q_{0}(n, n+1)+q^{n} Q_{1}(n, n)\right)+Q_{0}(n-1, n+1) \\
& \left.+q^{n} Q_{1}(n, n)+q^{n+1} Q_{1}(n, n+1)+Q_{0}(n+1, n+1)\right)
\end{aligned}
$$




$$
\begin{aligned}
&= Y_{2 n+1}+\left(q^{n+1}+q^{2 n+2}\right)\left(Y_{2 n-1}+Y_{2 n}\right)-q^{n+1} Q_{0}(n-1, n+1) \\
&-Q_{0}(n, n+1)-q^{n+1} Q_{0}(n, n+1)-q^{2 n+1} Q_{1}(n, n) \\
&+q^{n+1} Q_{1}(n, n+1)+Q_{0}(n+1, n+1) \\
&= Y_{2 n+1}+\left(q^{n+1}+q^{2 n+2}\right)\left(Y_{2 n-1}+Y_{2 n}\right)-q^{n+1} Q_{0}(n-1, n+1) \\
&-Q_{0}(n, n+1)-q^{2 n+1} Q_{1}(n, n)+Q_{0}(n+1, n+1)-q^{n+1}\left[\begin{array}{c}
2 n+1 \\
n
\end{array}\right] \\
&= Y_{2 n+1}+\left(q^{n+1}+q^{2 n+2}\right)\left(Y_{2 n-1}+Y_{2 n}\right)-q^{n+1} Q_{0}(n-1, n+1) \\
&-q^{2 n+1} Q_{1}(n, n)+q^{n+1} Q_{-1}(n-1, n+2)-q^{n+1}\left[\begin{array}{c}
2 n+1 \\
n
\end{array}\right] \\
&= Y_{2 n+1}+\left(q^{n+1}+q^{2 n+2}\right)\left(Y_{2 n-1}+Y_{2 n}\right) \\
&-q^{n+1} Q_{0}(n-1, n+1)-q^{2 n+1} Q_{1}(n, n) \\
&+q^{n+1}\left[\begin{array}{l}
2 n+1 \\
n+1
\end{array}+q^{n+1} Q_{0}(n-1, n+2)-q^{n+1}\left[\begin{array}{c}
2 n+1 \\
n
\end{array}\right]\right. \\
&= Y_{2 n+1}+\left(q^{n+1}+q^{2 n+2}\right)\left(Y_{2 n-1}+Y_{2 n}\right) \\
&+q^{n+1}\left(Q_{0}(n-1, n+2)-Q_{0}(n-1, n+1)-q^{n} Q_{1}(n, n)\right) \\
&= Y_{2 n+1}+\left(q^{n+1}+q^{2 n+2}\right)\left(Y_{2 n-1}+Y_{2 n}\right) \\
&= Y_{2 n+1}+\left(q^{n+1}+q^{2 n+2}\right)\left(Y_{2 n+1}+\left(1-q^{2 n+1}\right) Y_{2 n-1}\right) \\
& \text { (by }(2.16
\end{aligned}
$$

Thus the polynomials $Y_{n}$ satisfy the initial conditions $Y_{-1}=0, Y_{0}=1$ and (1.1) and (1.2). Consequently

$$
Y_{n}=\chi_{n}(0,1, q) \text { for all } n \geqslant-1 \text {. }
$$

Combining (2.23) with (2.19) and (2.20), we see that (2.6) and (2.7) are established.

\section{The limiting identities}

It should be pointed out that Wall polynomials possess other striking limits that are neither addressed in [2] and [3] nor are obviously amenable to the techniques developed there. In order to consider these new limits we must reverse the Wall polynomial. That is, define

$$
\begin{aligned}
& R_{2 n}(1,1, q)=q^{n^{2}+n} \chi_{2 n}\left(1,1, q^{-1}\right) \\
& R_{2 n-1}(1,1, q)=q^{n^{2}} \chi_{2 n-1}\left(1,1, q^{-1}\right)
\end{aligned}
$$




$$
\begin{gathered}
R_{2 n}(0,1, q)=q^{n^{2}+n} \chi_{2 n}\left(0,1, q^{-1}\right), \\
R_{2 n-1}(0,1, q)=q^{n^{2}-1} \chi_{2 n-1}\left(1,1, q^{-1}\right) .
\end{gathered}
$$

Inspection of the recurrence relations (1.1) and (1.2) shows that the $R_{n}$ 's are all polynomials in $q$ with nonzero constant term. Furthermore the empirical examination of extensions of Tables 1 and 2 leads one directly to conjecture the following results:

$$
\begin{aligned}
\lim _{n \rightarrow \infty} R_{2 n}(1,1, q) & =\frac{2 \sum_{j=-\infty}^{\infty} q^{3 j^{2}+2 j}}{\prod_{j=1}^{\infty}\left(1-q^{j}\right)} \\
& =2 \prod_{n=1}^{\infty} \frac{\left(1-q^{6 n}\right)\left(1+q^{2 n-1}\right)}{\left(1+q^{6 n-3}\right)\left(1-q^{n}\right)}
\end{aligned}
$$

$$
\begin{aligned}
\lim _{n \rightarrow \infty} R_{2 n-1}(1,1, q) & =\frac{\sum_{j=-\infty}^{\infty} q^{3 j^{2}}}{\prod_{j=1}^{\infty}\left(1-q^{j}\right)} \\
& =\prod_{n=1}^{\infty} \frac{\left(1-q^{6 n}\right)\left(1+q^{6 n-3}\right)^{2}}{\left(1-q^{n}\right)},
\end{aligned}
$$

$$
\begin{aligned}
\lim _{n \rightarrow \infty} R_{2 n}(0,1, q) & =\frac{\sum_{j=-\infty}^{\infty} q^{3 j^{2}+j}}{\prod_{j=1}^{\infty}\left(1-q^{j}\right)} \\
& =\prod_{n=1}^{\infty} \frac{\left(1-q^{6 n}\right)\left(1+q^{2 n}\right)}{\left(1+q^{6 n}\right)\left(1-q^{n}\right)},
\end{aligned}
$$

$$
\begin{aligned}
\lim _{n \rightarrow \infty} R_{2 n-1}(0,1, q) & =\frac{\sum_{j=1}^{\infty} q^{3 j^{2}+3 j}}{\prod_{n=1}^{\infty}\left(1-q^{n}\right)} \\
& =\prod_{n=1}^{\infty} \frac{\left(1-q^{6 n}\right)}{\left(1-q^{6 n-3}\right)\left(1-q^{n}\right)}
\end{aligned}
$$

In order to deduce the six limiting identities we need only two observations: First, for fixed integers $a$ and $b$ :

$$
\lim _{n \rightarrow \infty}\left[\begin{array}{c}
2 n+a \\
n+b
\end{array}\right]=\prod_{n=1}^{\infty} \frac{1}{1-q^{n}} .
$$

Second if $q$ is replaced by $q^{-1}$ in the Gaussian polynomial $\left[{ }_{B}^{A}\right]$, the result is

$$
q^{-B(A-B)}\left[\begin{array}{l}
A \\
B
\end{array}\right]
$$


No difficulties arise when we pass limits inside the summation signs as long as $|q|<1$ for then the quadratic exponent on $q$ dominates all convergence questions.

Proof OF (1.3). Clearly by (1.1) $\chi_{2 n}$ and $\chi_{2 n+1}$ converge to the same limit (if any). Hence

$$
\begin{aligned}
\chi(1,1, q) & =\lim _{n \rightarrow \infty} \chi_{2 n-1}(1,1, q)=\lim _{n \rightarrow \infty} \sum_{j=-\infty}^{\infty}\left[\begin{array}{c}
2 n \\
n+2 j
\end{array}\right] q^{j^{2}} \\
& =\frac{\sum_{j=-\infty}^{\infty} q^{j^{2}}}{\prod_{n=1}^{\infty}\left(1-q^{n}\right)} \quad(\text { by }(2.4)) .
\end{aligned}
$$

Proof of (1.4). As above

$$
\begin{aligned}
\chi(0,1, q) & =\lim _{n \rightarrow \infty} \chi_{2 n-1}(0,1, q)=\lim _{n \rightarrow \infty} \sum_{j=0}^{\infty}\left[\begin{array}{c}
2 n \\
n-1-j
\end{array}\right] q^{j^{2}+j} \\
& =\frac{\sum_{j=0}^{\infty} q^{j^{2}+j}}{\prod_{n-1}^{\infty}\left(1-q^{n}\right)} \quad(\text { by }(2.6)) .
\end{aligned}
$$

Proof of (3.5).

$$
\begin{aligned}
& \lim _{n \rightarrow \infty} R_{2 n}(1,1, q)=\lim _{n \rightarrow \infty} q^{n^{2}+n} \chi_{2 n}\left(1,1, q^{-1}\right) \\
&=\lim _{n \rightarrow \infty} q^{n^{2}+n}\left(\sum _ { j = - \infty } ^ { \infty } \left(q^{-(n+2 j+1)(n-2 j)}\left[\begin{array}{c}
2 n+1 \\
n+2 j+1
\end{array}\right]\right.\right. \\
&\left.\left.\quad+q^{-n-(n+2 j+1)(n-2 j-1)}\left[\begin{array}{c}
2 n \\
n+2 j+1
\end{array}\right]\right) q^{-j^{2}}\right) \\
&=\lim _{n \rightarrow \infty} \sum_{j=-\infty}^{\infty}\left(\left[\begin{array}{c}
2 n+1 \\
n+2 j+1
\end{array}\right] q^{3 j^{2}+2 j}+\left[\begin{array}{c}
2 n \\
n+2 j+1
\end{array}\right] q^{3 j^{2}+4 j+1}\right) \\
&=\frac{\left(\sum_{j=-\infty}^{\infty} q^{3 j^{2}+2 j}+\sum_{j=-\infty}^{\infty} q^{\left.3 j^{2}+4 j+1\right)}\right.}{\prod_{n=1}^{\infty}\left(1-q^{n}\right)} \\
&=\frac{2 \sum_{j=-\infty}^{\infty} q^{3 j^{2}+2 j}}{\prod_{n=1}^{\infty}\left(1-q^{n}\right)} \quad \text { (where } j \rightarrow-j-1 \text { in second sum). }
\end{aligned}
$$

The second portion of (3.5) follows directly from an application of Jacobi's triple product identity [1, page 21$]$ to the numerator above.

Identities (3.6), (3.7) and (3.8) follow in precisely this manner. 


\section{Conclusion}

The most obvious questions arising from this work are:

1. Is there a reasonable combinatorial interpretation of Theorem 1? In particular can the "lattice path" setting of Garsia and Remmel [3] be used to prove Theorem 1?

2. Are equations (3.5)-(3.8) relevant in group theory? As previously noted, (1.3) and (1.4) are.

3. Since

$$
\begin{aligned}
\sum_{n, m>0} \frac{P(n, m) t^{n} u^{m}}{(1-q)\left(1-q^{2}\right) \cdots\left(1-q^{n+m}\right)} \\
=\prod_{n=0}^{\infty} \frac{\left(1-q^{2 n+2}\right)\left(1+t^{2} u^{-2} q^{2 n+1}\right)\left(1+u^{2} t^{-2} q^{2 n+1}\right)}{\left(1-u q^{n}\right)\left(1-t q^{n}\right)}
\end{aligned}
$$

can one deduce properties of the $\chi_{n}(1,1, q)$ from this result?

\section{References}

[1] G. E. Andrews, 'The theory of partitions,' Encyclopedia of mathematics and its applications, Vol. 2 (Addison-Wesley, Reading, Mass., 1976).

[2] G. E. Andrews, 'Partitions, $q$-series, and the Lusztig-Macdonald-Wall conjectures,' Invent. Math. 41 (1977), 91-102.

[3] A. Garsia and J. Remmel, 'A combinatorial view of Andrews' proof of the L-M-W conjectures,' European J. Combin., to appear.

[4] G. Lusztig, 'Irreducible representations of finite classical groups,' Invent. Math. 43 (1977), 125-177.

[5] G. E. Wall, 'On the conjugacy classes in the unitary, symplectic and orthogonal groups,' $J$. Austral. Math. Soc. 3 (1963), 1-62.

The Pennsylvania State University

University Park, Pennsylvania

U.S.A. 